\title{
An Analysis of Database Workload Performance on Simultaneous Multithreaded Processors
}

\author{
Jack L. Lo, Luiz André Barroso*, Susan J. Eggers, Kourosh Gharachorloo*, \\ Henry M. Levy, and Sujay S. Parekh \\ Dept. of Computer Science and Engineering \\ Box 352350 \\ University of Washington \\ Seattle, WA 98195 \\ * Digital Equipment Corporation \\ Western Research Laboratory \\ 250 University Ave. \\ Palo Alto, CA 94301
}

\begin{abstract}
Simultaneous multithreading (SMT) is an architectural technique in which the processor issues multiple instructions from multiple threads each cycle. While SMT has been shown to be effective on scientific workloads, its performance on database systems is still an open question. In particular, database systems have poor cache performance, and the addition of multithreading has the potential to exacerbate cache conflicts.

This paper examines database performance on SMT processors using traces of the Oracle database management system. Our research makes three contributions. First, it characterizes the memory-system behavior of database systems running on-line transaction processing and decision support system workloads. Our data show that while DBMS workloads have large memory footprints, there is substantial data reuse in a small, cacheable "critical" working set. Second, we show that the additional data cache conflicts caused by simultaneousmultithreaded instruction scheduling can be nearly eliminated by the proper choice of software-directed policies for virtual-to-physical page mapping and per-process address offsetting. Our results demonstrate that with the best policy choices, D-cache miss rates on an 8-context SMT are roughly equivalent to those on a single-threaded superscalar. Multithreading also leads to better interthread instruction cache sharing, reducing I-cache miss rates by up to $35 \%$. Third, we show that SMT's latency tolerance is highly effective for database applications. For example, using a memory-intensive OLTP workload, an 8context SMT processor achieves a 3-fold increase in instruction throughput over a single-threaded superscalar with similar resources.
\end{abstract}

\section{Introduction}

With the growing importance of internet commerce, data mining, and various types of information gathering and processing, database systems will assume an even more crucial role in computer systems of the future from the desktop to highly-scalable multiprocessors or clusters. Despite their increasing prominence, however, database management systems (DBMS) have been the subject of only limited architectural study [3,6,12,16,22]. Not surprisingly, these studies have shown that database systems can exhibit strikingly high cache miss rates. In the past, these miss rates were less significant, because $\mathrm{I} / \mathrm{O}$ latency was the limiting factor for database performance. However, with the latest generation of commercial database engines employing numerous processes, disk arrays, increased I/O concurrency, and huge memories, many of the I/O limitations have been addressed [7]. Memory system performance is now the crucial problem: the high miss rates of database workloads, coupled with long memory latencies, make the design of future CPUs for database execution a significant challenge.

This paper examines the memory system behavior of database management systems on simultaneous multithreaded processors. Simultaneous multithreading (SMT) [4] is an architectural technique in which the processor issues instructions from multiple threads in a single cycle. For scientific workloads, SMT has been shown to substantially increase processor utilization through finegrained sharing of all processor resources (the fetch and issue logic, the caches, the TLBs, and the functional units) among the executing threads [23]. However, SMT performance on commercial databases is still an open research question, and is of interest for three related reasons. First, a database workload is intrinsically multithreaded, providing a natural source of threads for an SMT processor. Second, many database workloads are memory-intensive and lead to extremely low processor utilization. For example, our studies show that a transaction processing workload achieves only 0.79 IPC on an 8wide, out-of-order superscalar with $128 \mathrm{~KB} \mathrm{L1}$ caches less than 1/4 the throughput of the SPEC suite. As a result, there is great potential for increased utilization through simultaneous multithreaded instruction issue. Third, but somewhat troubling, SMT's fine-grained shar- 
ing of the caches among multiple threads may seriously diminish memory system performance, because database workloads can stress the cache to begin with even on a single-threaded superscalar. Therefore, while SMT seems a promising candidate to address the low instruction throughput on database systems, the memory system behavior of databases presents a potentially serious challenge to the multithreaded design approach. That challenge is the focus of this paper.

To investigate database memory system behavior on SMT processors, we have instrumented and measured the Oracle version 7.3.2 database system executing under Digital UNIX on DEC Alpha processors. We use traces of on-line transaction processing (OLTP) and decision support system (DSS) workloads to drive a highlydetailed trace-driven simulator for an 8-context, 8-wide simultaneous multithreaded processor. Our analysis of the workload goes beyond previous database memory system measurements to show the different memory access patterns of a DBMS's internal memory regions (instruction segment, private data, database buffer cache, and shared metadata) and the implications those patterns have for SMT memory system design.

Our results show that while cache interference among competing threads can be significant, the causes of this interference can often be mitigated with simple software policies. For example, we demonstrate a substantial improvement in IPC for the OLTP workload through the selection of an appropriate virtual-to-physical page mapping algorithm in the operating system. We also show that some of the inter-thread memory-system competition is constructive, i.e., the sharing of data among threads leads to cache-line reuse, which aids SMT performance. Overall, we demonstrate that simultaneous multithreading can tolerate memory latencies, exploit inter-thread instruction sharing, and limit inter-thread interference on memory-intensive database workloads. On the highly memory-intensive OLTP workload, for example, our simulated SMT processor achieves a 3-fold improvement in instruction throughput over a base superscalar design with similar resources.

The organization of the paper follows the approach described above. Section 2 describes the methodology used in our simulation-based study. Section 3 characterizes the memory behavior of on-line transaction processing and decision support system workloads, motivating the use of SMT as a latency-tolerance technique. Section 4 quantifies the effect of constructive and destructive cache interference in both the instruction and data caches and evaluates alternatives for reducing interthread conflict misses. Section 5 compares the performance of the OLTP and DSS workloads on SMT and a wide-issue superscalar, explaining the architecture basis for SMT's higher instruction throughput. Finally, we discuss related work and conclude.

\begin{tabular}{|l||l|}
\hline Functional units & 6 integer (including 4 ld/st units), 4 FP \\
\hline Instruction queue & 32 integer entries, 32 FP entries \\
\hline Active list & 128 entries/context \\
\hline Architectural registers & $32 * 8$ integer / 32*8 FP \\
\hline Renaming registers & 100 integer / 100 FP \\
\hline Instruction retirement & up to 12 instructions per cycle \\
\hline
\end{tabular}

Table 1: CPU parameters used in our simulator. The instruction window size is limited by both the active list and the number of renaming registers.

\section{Methodology}

This section describes the methodology used for our experiments. We begin by presenting details of the hardware model implemented by our trace-driven processor simulator. We then describe the workload used to generate traces and our model for the general execution environment of database workloads.

\subsection{SMT processor model}

Simultaneous multithreading exploits both instructionlevel and thread-level parallelism by executing instructions from multiple threads each cycle. This combination of wide-issue superscalar technology and fine-grain hardware multithreading improves utilization of processor resources, and therefore increases instruction throughput and program speedups. Previous research has shown that an SMT processor can be implemented with rather straightforward modifications to a standard dynamically-scheduled superscalar [23].

Our simulated SMT processor is an extension of a modern out-of-order, superscalar architecture, such as the MIPS R10000. During each cycle, the SMT processor fetches eight instructions from up to two of the eight hardware contexts. After instructions are decoded, register renaming removes false register dependencies both within a thread (as in a conventional superscalar) and between threads, by mapping context-specific architectural registers onto a pool of physical registers. Instructions are then dispatched to the integer or floatingpoint instruction queues. The processor issues instructions whose register operands have been computed; ready instructions from any thread may issue any cycle. Finally, the processor retires completed instructions in program order.

To support simultaneous multithreading, the processor replicates several resources: state for hardware contexts (registers and program counters) and per-context mechanisms for pipeline flushing, instruction retirement, trapping, precise interrupts, and subroutine return prediction. In addition, the branch target buffer and translation lookaside buffer contain per-context identifiers.

Table 1 provides more details describing our processor model, and Table 2 lists the memory system parameters. Branch prediction uses a McFarling-style, hybrid branch predictor [13] with an 8K-entry global pre- 


\begin{tabular}{|l||r|r|r|}
\hline & L1 I-cache & L1 D-cache & L2 cache \\
\hline \hline Size & 128KB & 128KB & 16MB \\
\hline Line size & $64 \mathrm{~B}$ & $64 \mathrm{~B}$ & $64 \mathrm{~B}$ \\
\hline $\begin{array}{l}\text { Miss latency to next } \\
\text { level (cycles) }\end{array}$ & 10 & 10 & 68 \\
\hline Associativity & 2-way & 2-way & direct-mapped \\
\hline Fill latency (cycles) & 2 & 2 & 4 \\
\hline Banks & 4 & 4 & 1 \\
\hline Ports/bank & 1 & 2 & 1 \\
\hline Max. in-flight misses & 16 & 16 & 16 \\
\hline
\end{tabular}

Table 2: Memory system parameters used in our simulator. The instruction and data TLBs are both 128-entry and fullyassociative, with 20 cycle miss penalties.

diction table, a $2 \mathrm{~K}$-entry local history table which indexes into a $4 \mathrm{~K}$-entry local prediction table, and an $8 \mathrm{~K}$ entry selection table to choose between the local and global predictors.

\subsection{Simulating database workloads}

Compared to typical benchmarks, such as SPEC and SPLASH, commercial workloads have substantially more complex execution behavior. Accurate simulation of these applications must capture this complexity, especially I/O latencies and the interaction of the database with the operating system. We therefore examined the behavior of the Oracle DBMS and the underlying Digital UNIX operating system to validate and strengthen our simulation methodology. Though DBMS source code was not available, we used both the Digital Continuous Profiling Infrastructure (DCPI) [1] and separate experiments running natively on Digital AlphaServers to understand DBMS behavior and extract appropriate parameters for our simulations. The remainder of this section describes the experimental methodology, including the workloads, trace generation, operating system activity (including modelling of $\mathrm{I} / \mathrm{O}$ ), and synchronization.

\section{The database workload}

On-line transaction processing (OLTP) and decision support systems (DSS) dominate the workloads handled by database servers; our studies use two workloads, one representative of each of these domains. Our OLTP workload is based on the TPC-B benchmark [20]. Although TPC-C has supplanted TPC-B as TPC's current OLTP benchmark, we found that the two workloads have similar processor and memory system characteristics [2]. We chose TPC-B because it is easier to set up and run.

The OLTP workload models transaction processing for a bank, where each transaction corresponds to a bank account deposit. Each transaction is small, but updates several database tables (e.g., teller and branch). OLTP workloads are intrinsically parallel, and therefore database systems typically employ multiple server processes to process client transactions and hide I/O latencies.

In decision support systems, queries execute against a large database to answer critical business questions. The database consists of several inter-related tables, such as parts, nations, customers, orders, and lineitems. Our DSS workload is based on query 6 of the TPC-D benchmark [21], which models the database activity for a business that manages, sells, or distributes products worldwide. The query scans the largest table (lineitem) to quantify the amount of revenue increase that would have resulted from eliminating certain discounts in a given percentage range in a given year. This query is representative of DSS workloads; other TPC-D queries tend to have similar memory system behavior [2].

\section{Trace generation}

Commercial database applications require considerable tuning to achieve optimal performance. Because the execution time of different workload components (user, kernel, I/O, etc.) may vary depending on this level of optimization and customization, we extensively tuned Oracle v.7.3.2 and Digital UNIX to maximize database performance when running natively on a 4-processor Digital AlphaServer 4100. Using the best-performing configuration, we instrumented the database application with ATOM [17] and generated a separate instruction trace file for each server process. We then fed these traces to our cycle-level SMT simulator, whose parameters were described above. In each experiment, our workload consists of 16 processes (threads), unless otherwise noted. For the OLTP workload, each process contains 315 transactions (a total of 5040) on a 900MB database. For a single OLTP experiment, we simulate roughly 900M instructions. For our DSS workload, scaling is more complex, because the run time (and therefore, simulation time) grows linearly with the size of the database. Fortunately, the DSS query exhibits very consistent behavior throughout its execution, so we could generate representative traces using sampling techniques [2]. With the sampled traces, each of our DSS experiments simulate roughly $500 \mathrm{M}$ instructions from queries on a $500 \mathrm{MB}$ database.

\section{Operating system activity}

Although ATOM generates only user-level traces, we took several measures to ensure that we carefully modelled operating system effects. While some previous studies have found that operating system kernel activity can dominate execution time for OLTP workloads $[6,12$, 16], we found that a well-tuned workload spends most of its time in user-level code. Using DCPI, we determined that for OLTP, roughly $70 \%$ of execution time was spent in user-level code, with the rest in the kernel and the idle loop. For DSS, kernel and idle time were negligible. These measurements therefore verified that our traces account for the dominant database activity. 
In addition, we monitored the behavior of Digital UNIX to ensure that our simulation framework models the behavior of the operating system scheduler and underlying I/O subsystem to account for I/O latencies. We use a simple thread scheduler when there are more processes (threads) than hardware contexts. Although the scheduler can preempt threads at the end of a $500 \mathrm{~K}$-cycle scheduling quantum, most of the scheduling decisions are guided by hints from the server processes via four UNIX system calls: fread, fwrite, pid_block, and pid_unblock. We therefore annotate the traces to indicate where the server processes call these routines.

The OLTP workload uses fread and fwrite calls for pipe communication between the client (the application) and the server process. Writes are non-blocking, while reads have an average latency of 14,500 cycles on the AlphaServer. Our simulator models this fread latency and treats both fread and fwrite as hints to the scheduler to yield the processor. The other important system call, pid_block, is primarily used during the commit phase of each transaction. During transaction commit, the logwriter process must write to the $\log$ file. The pid_block call is another scheduler hint that yields the CPU to allow the logwriter to run more promptly.

For our DSS workload, system calls are infrequent, but the server processes periodically invoke freads to bring in new $128 \mathrm{~KB}$ database blocks for processing.

Our simulation experiments also include the impact of the I/O subsystem. For the OLTP workload, we use a $1 \mathrm{M}$ cycle latency (e.g., $1 \mathrm{~ms}$ for a $1 \mathrm{GHz}$ processor) for the logwriter's small (about $8 \mathrm{~KB}$ ) file writes. This latency models a fast I/O subsystem with non-volatile RAM to improve the performance of short writes. For DSS, we model database reads (about $128 \mathrm{~KB}$ ) with $5 \mathrm{M}$ cycle latencies. Most of our experiments use 16 processes, but in systems with longer I/O latencies, more processes will be required to hide $\mathrm{I} / \mathrm{O}$.

\section{Synchronization}

Oracle's primary synchronization primitive uses the Alpha's load-locked/store-conditional instructions, and higher-level locks are built upon this mechanism. However, on an SMT processor, this conventional spinning synchronization can have adverse effects on threads running in other contexts, because the spinning instructions consume processor resources that could be used more effectively by the other threads. We therefore use hardware blocking locks, which are a more efficient synchronization mechanism for SMT processors. To incorporate blocking synchronization in the simulations, we replaced the DBMS's synchronization scheme with blocking locks in the traces.

\section{Database workload characterization}

This section characterizes the memory-system behavior of our commercial OLTP and DSS workloads, providing a basis for the detailed SMT architectural simulations presented in Section 4. While previous work has shown that high miss rates can be generated by commercial workloads, we go beyond that observation to uncover the memory-access patterns that lead to the high miss rates.

A database's poor memory system performance causes a substantial instruction throughput bottleneck. For example, our processor simulations (described in the next section) show that the OLTP workload achieves only 0.79 instructions per cycle on an 8-wide, singlethreaded, superscalar with $128 \mathrm{~KB} \mathrm{L1}$ caches (compared to 3.3 IPC for a subset of SPEC benchmarks on the same processor). The OLTP workload achieves only 0.26 IPC with 32KB L1 caches! Because of its latency-hiding capability, simultaneous multithreading has the potential to substantially improve the single-threaded superscalar's low IPC. On the other hand, SMT could exacerbate conflicts in the already-overloaded caches beyond its ability to hide the latencies. An evaluation of this issue requires an analysis of the thread working sets, their access patterns, and the amount of inter-thread sharing. We provide that analysis in this section.

Our studies of memory-system behavior focus on the performance of the database server processes that dominate execution time for commercial workloads. In Oracle's dedicated mode, a separate server process is associated with each client process. Each server process accesses memory in one of 3 segments:

- The instruction text segment contains the database code and is shared among all database processes.

- The Program Global Area (PGA) contains perprocess data, such as private stacks, local variables, and private session variables.

- The Shared Global Area (SGA) contains the database buffer cache, the data dictionary (indices and other metadata), the shared SQL area (which allows multiple users to share a single copy of an SQL statement), redo logs (for tracking data updates and guiding crash recovery), and other shared resources. The SGA is the largest region and is shared by all server processes. For the purposes of this study, we consider the database buffer cache to be a fourth region (which we'll call the SGA buffer cache), separate from the rest of the SGA (called $S G A$-other), because its memory access pattern is quite distinct.

To better understand memory behavior, we compare and analyze the memory access patterns of these regions on both OLTP and DSS workloads.

\subsection{OLTP characterization}

As described in the previous section, we traced our OLTP workload, which models transaction processing for a bank. We then used these traces to analyze cache 


\begin{tabular}{|c|c|c|c|c|c|c|c|c|c|c|c|c|}
\hline \multirow{3}{*}{$\begin{array}{l}\text { Program } \\
\text { Segments }\end{array}$} & \multicolumn{6}{|c|}{ OLTP } & \multicolumn{6}{|c|}{ DSS } \\
\hline & \multicolumn{2}{|c|}{ L1 cache miss rate } & \multirow{2}{*}{$\begin{array}{l}\text { Memory } \\
\text { footprint }\end{array}$} & \multirow{2}{*}{$\begin{array}{l}\text { Avg. \# of } \\
\text { refs per } \\
\text { 64-byte } \\
\text { block }\end{array}$} & \multicolumn{2}{|c|}{$\begin{array}{c}\text { Avg. \# accesses to a } \\
\text { block until a cache } \\
\text { conflict }\end{array}$} & \multicolumn{2}{|c|}{ L1 cache miss rate } & \multirow{2}{*}{$\begin{array}{l}\text { Memory } \\
\text { footprint } \\
\text { (sample) }\end{array}$} & \multirow{2}{*}{$\begin{array}{l}\text { Avg. \# of } \\
\text { refs per } \\
\text { 64-byte } \\
\text { block }\end{array}$} & \multicolumn{2}{|c|}{$\begin{array}{l}\text { Avg. \# accesses to a } \\
\text { block until a cache } \\
\text { conflict }\end{array}$} \\
\hline & $32 \mathrm{~KB}$ & $128 \mathrm{~KB}$ & & & $32 \mathrm{~KB}$ & $128 \mathrm{~KB}$ & $32 \mathrm{~KB}$ & $128 \mathrm{~KB}$ & & & $32 \mathrm{~KB}$ & $128 \mathrm{~KB}$ \\
\hline Instruction & $23.3 \%$ & $13.7 \%$ & 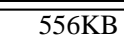 & 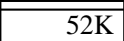 & $\overline{3}$ & 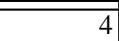 & $\overline{0.5 \%}$ & $0.0 \%$ & 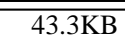 & $216 \mathrm{~K}$ & $\overline{11}$ & $\overline{\overline{43}}$ \\
\hline PGA & $8.4 \%$ & $7.4 \%$ & $1.3 \mathrm{MB}$ & $14 \mathrm{~K}$ & 8 & 11 & $0.8 \%$ & $0.7 \%$ & $2.2 \mathrm{MB}$ & $3.8 \mathrm{~K}$ & $\overline{38}$ & 102 \\
\hline $\begin{array}{l}\text { SGA buffer } \\
\text { cache }\end{array}$ & $7.5 \%$ & $6.8 \%$ & $9.3 \mathrm{MB}$ & 66 & 9 & 12 & $9.3 \%$ & $8.0 \%$ & $2.7 \mathrm{MB}$ & 383 & 7 & 10 \\
\hline SGA-other & $17.5 \%$ & $12.9 \%$ & $26.5 \mathrm{MB}$ & 169 & 3 & 5 & $0.4 \%$ & $0.2 \%$ & $878 \mathrm{~KB}$ & $3.4 \mathrm{~K}$ & 43 & 117 \\
\hline $\begin{array}{l}\text { All data seg- } \\
\text { ments }\end{array}$ & $10.1 \%$ & $8.4 \%$ & $37.1 \mathrm{MB}$ & 630 & 7 & 9 & $1.5 \%$ & $1.2 \%$ & $5.8 \mathrm{MB}$ & $2.8 \mathrm{~K}$ & 29 & 59 \\
\hline
\end{tabular}

Table 3: Memory behavior characterization for OLTP (16 processes, 315 transactions each) and DSS (16 processes) on a single-threaded uniprocessor. The characterization for only 8 processes (a typical number for hiding $1 / O$ on existing processors) is qualitatively the same (results not shown). Footprints are smaller, but the miss rates are comparable. On the uniprocessor, 16 processes only degraded L1 cache miss rates by 1.3 percentage points for the OLTP workload, when compared to 8 processes. Results are shown for both 32KB and 128KB caches. All caches are 2-way associative.

behavior for a traditional, single-threaded uniprocessor. The left-hand side of Table 3 shows our results for the OLTP workload (we discuss the DSS results later). Overall, this data confirms the aggregate cache behavior of transaction processing workloads found by others; namely, that they suffer from higher miss rates than scientific codes (at least as exhibited by SPEC and SPLASH benchmarks), with instruction misses a particular problem $[3,6,12,16]$. For example, columns 2 and 3 of Table 3 show that on-chip caches are relatively ineffective both at current cache sizes $(32 \mathrm{~KB})$ and at larger sizes $(128 \mathrm{~KB})$ expected in next-generation processors. In addition, instruction cache behavior is worse than data cache behavior, having miss rates of $23.3 \%$ and $13.7 \%$ for $32 \mathrm{~K}$ and $128 \mathrm{~K}$ caches, respectively. (Note, however, that the instruction cache miss rate is computed by dividing the number of misses by the number of I-cache fetches, not by the number of instructions. In our experiments, a single I-cache access can fetch up to 8 instructions.)

In more detail, Table 3 shows a breakdown of cacheaccess information by memory region. Here we see that the high miss rates are partly attributable to OLTP's large memory footprints, which range from 556KB in the instruction segment up to $26.5 \mathrm{MB}$ in SGA-other. The footprints for all four regions easily exceed on-chip cache sizes; for the two SGA areas, even large off-chip caches are insufficient.

Surprisingly, the high miss rates are not a consequence of a lack of instruction and data reuse. Column 5 shows that, on average, blocks are referenced very frequently, particularly in the PGA and instruction regions. Cache reuse correlates strongly with the increase in the memory footprint size as transactions are processed. For example, our data (not shown) indicates that as more of the database is accessed, the memory footprint of the
SGA buffer cache continues to grow and exceeds that of the SGA-other, whose size levels off over time; reuse in the buffer cache is therefore relatively low. In contrast, the PGA and instruction segment footprints remain fairly stable over time, and reuse is considerably larger in those regions.

High reuse only reduces miss rates, however, if multiple accesses to cache blocks occur over a short enough period of time that the blocks are still cache-resident. Results in columns 6 and 7 show that the frequency of block replacement strongly and inversely correlates with miss rates, for all segments. Replacement is particularly frequent in the instruction segment, where cache blocks are accessed on average only 3 or 4 times before they are potentially replaced ${ }^{1}$, either by a block from this thread or another thread. So, despite a relatively small memory footprint and high reuse, the instruction segment's miss rate is high.

In summary, all three of these factors, large memory footprints, frequency of memory reuse, and the interval length between cache conflicts, make on-chip caching for OLTP relatively ineffective.

\section{The "critical" working set}

Within a segment, cache reuse is not uniformly distributed across blocks, and for some segments is highly skewed, a fact hidden by the averaged data in Table 3. To visualize this, Figure 1 characterizes reuse in the four memory regions. To obtain data points for these graphs, we divided the memory space into 64-byte (cache-line sized) blocks and calculated how many times each was

1. Columns 6 and 7 measure inherent cache mapping conflicts using a direct-mapped, instead of two-way associative, cache. Even though this may overestimate the number of replacements (compared to two-way), the relative behavior for the different data segments is still accurate. 

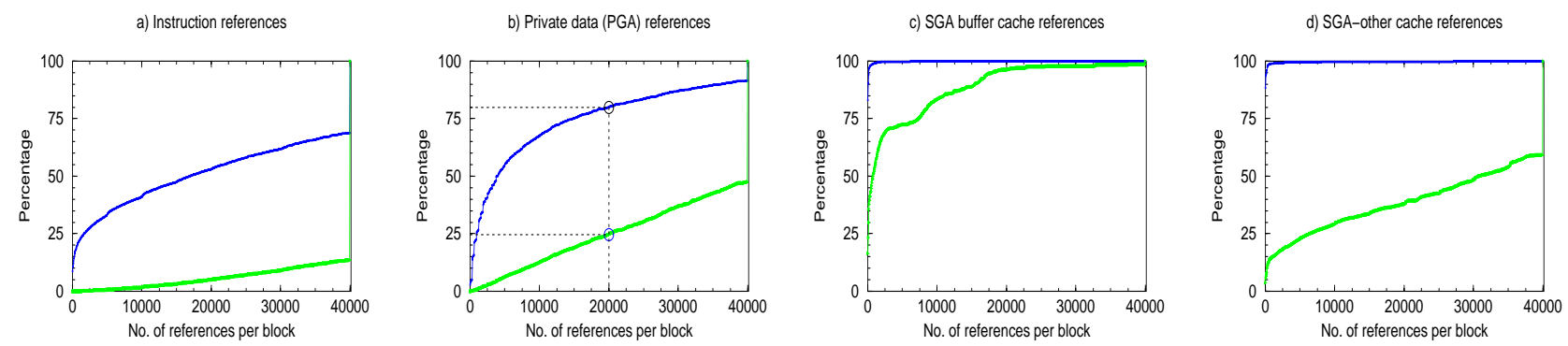

Figure 1. OLTP locality profiles. In each graph, the upper curve plots the cumulative percentage of 64-byte blocks accessed $\mathrm{n}$ times or less; the lower graph plots the cumulative percentage of references made to blocks accessed $\mathrm{n}$ times or less.
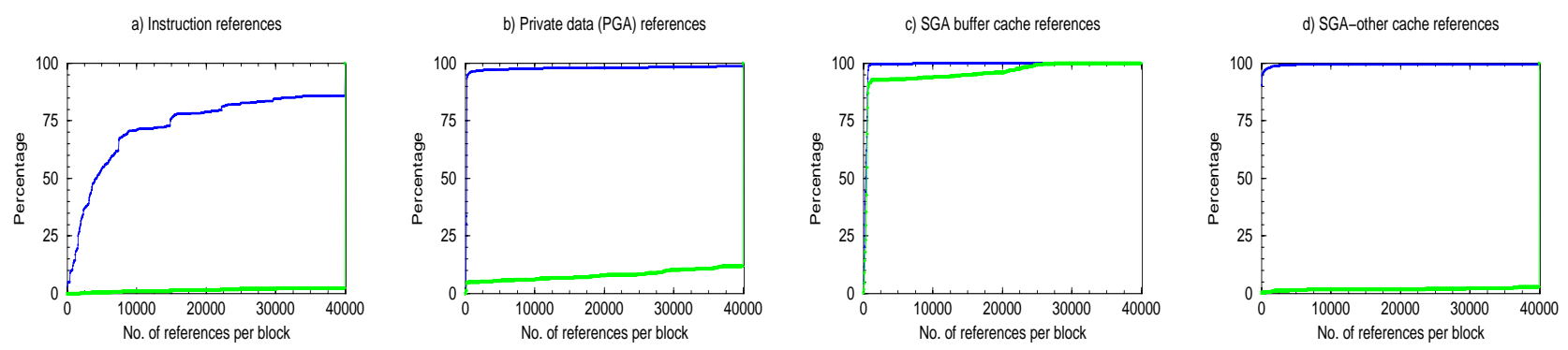

Figure 2. DSS locality profiles.

accessed. The black line (the higher of the two lines) plots a cumulative histogram of the percentage of blocks that are accessed $\mathrm{n}$ times or less; for example, the top circle in Figure 1b says that for the PGA, $80 \%$ of the blocks are accessed 20,000 times or less. The gray line (bottom) is a cumulative histogram that plots the percentage of total references that occurred to blocks accessed $\mathrm{n}$ times or less; the lower circle in Figure 1b shows that those blocks accessed 20,000 times or less account for only $25 \%$ of total references. Alternatively, these two points indicate that $20 \%$ of the blocks are accessed more than 20,000 times and account for $75 \%$ of all the references. In other words, for the PGA, a minority of the memory blocks are responsible for most of the memory references. (The curves in Figure 1 are all cumulative distributions and thus reach $100 \%$; we have omitted part of the right side of the graphs for most cases because the curves have long tails.)

All four regions exhibit skewed reference distributions, but to different extents. Comparing them at the highest reuse data point plotted in Figure 1, i.e., more than $40 \mathrm{~K}$ accesses per block, $31 \%$ of the blocks in the instruction segment account for $87 \%$ of the instruction references (Figure 1a), $8.5 \%$ of the blocks in the PGA account for $53 \%$ of the references (Figure 1b), and a remarkable $0.1 \%$ of the blocks in SGA-other account for $41 \%$ of the references (Figure 1d). The SGA buffer cache's reference distribution is also skewed (9\% of the blocks comprise $77 \%$ of the references); however, this point occurs at only 100 accesses. Consequently, most blocks in the SGA buffer cache $(91 \%)$ have very little reuse and the more frequently used blocks comprise a small percentage of total references.
Reference behavior that is skewed to this extent strongly implies that the "critical" working set of each segment, i.e., the portion of the segment that absorbs the majority of the memory references, is much smaller than the segment's memory footprint. As an example, the SGA-other blocks mentioned above are three orders of magnitude smaller (26KB) than this segment's memory footprint (26.5MB). The implication for simultaneous multithreading is that, for the segments that exhibit skewed reference behavior and make most of their references to a small number of blocks (instruction, PGA, and SGA-other segments), there will be some performancecritical portion of their working sets that fit comfortably into SMT's context-shared caches.

\subsection{DSS workload characterization}

As with OLTP, we used traces of the DSS workload to drive a simulator for a single-threaded uniprocessor. Our results, shown on the right half of Table 3, indicate that the DSS workload should cause fewer conflicts in the context-shared SMT caches than OLTP, because its miss ratios are lower, reuse is more clustered, and the segments' critical working sets are smaller. The instruction and (overall) data cache miss rates, as well as those of 2 of the 3 data segments (columns 8 and 9 of Table 3), are negligible, and cache reuse per block (columns 12 and 13 ) is sometimes even an order of magnitude higher. Because of more extreme reference skewing and/or smaller memory footprints, the cache-critical working sets for all segments except the SGA buffer cache are easily cacheable on an SMT. In the instruction region, 98\% of the references are made to only $6 \mathrm{~KB}$ of instruction text (Figure 2); and 253 blocks (16KB) account for $75 \%$ of 
PGA references. SGA-other is even more skewed, with more than $97 \%$ of the references touching only 51 blocks or $3 \mathrm{~KB}$.

The SGA buffer cache has a much higher miss rate than the other segments $(8 \%)$, because the query scans through the large lineitem table and little reuse occurs. The buffer cache is so uniformly accessed that its critical working set and memory footprint are almost synonymous; $99 \%$ of the blocks are touched fewer than 800 times, as shown by the locality histogram in Figure 2c.

The scalability of DSS's locality profile is an important issue as databases for decision support systems continue to grow in size. The reuse profiles demonstrate that the locality and good cache behavior in this workload scales to much larger databases. With larger databases (and therefore, longer-running queries), the instruction and PGA references dominate, but their working sets should remain small and easily cacheable. Although the footprints of both SGA segments grow with larger databases, DSS has good spatial locality independent of the size of the cache, and therefore references to these regions have minimal effects on locality.

\subsection{Summary of the workload characterization}

This section analyzed the memory-system behavior of the OLTP and DSS workloads in detail. Overall, we find that while the footprints (particularly for OLTP) are large for the various memory regions, there is good temporal locality in the most frequently accessed blocks, i.e., a small percentage of blocks account for most of the references. Thus, it is possible that even with multithreading, the "critical" working sets will fit in the caches, reducing the degradation on cache performance due to inter-thread conflicts.

Recall, however, that simultaneous multithreading interleaves per-thread cache accesses more finely than a single-threaded uniprocessor. Thus, inter-thread competition for cache lines will rise on an SMT, causing consecutive, per-thread block reuse to decline. If crossthread accesses are made to distinct addresses, increasing inter-thread conflicts, SMT will have to exploit temporal locality more effectively than the uniprocessor. But if the accesses occur to thread-shared blocks, inter-thread conflicts and misses will decline. The latter should be particularly beneficial for the instruction segment, where the various threads tend to execute similar code.

In the next section, we explore these implications, using a detailed simulation of an SMT processor executing the OLTP and DSS workloads.

\section{Multi-thread cache interference}

This section quantifies and analyzes the cache effects of OLTP and DSS workloads on simultaneous multithreaded processors. On conventional (single-threaded) processors, a DBMS employs multiple server processes to hide I/O latencies in the workload. Context switching between these processes may cause cache interference (i.e., conflicts), as blocks from a newly-scheduled process evict useful cache blocks from descheduled processes; however, once a thread begins to execute, it has exclusive control of the cache for the duration of its execution quantum. With simultaneous multithreading, thread execution is interleaved at a much finer granularity (within a cycle, rather than at the coarser contextswitch level). This fine-grained, simultaneous sharing of the cache potentially changes the nature of inter-thread cache interference. Understanding this interference is therefore key to understanding the performance of database workloads on SMT.

In the following subsections we identify two types of cache interference: destructive interference occurs when one thread's data replaces another thread's data in the cache, resulting in an increase in inter-thread conflict misses; constructive interference occurs when data loaded by one thread is accessed by another simultaneously-scheduled thread, resulting in fewer misses. We examine the effects of both destructive and constructive cache interference when running OLTP and DSS workloads on an SMT processor, and evaluate operating system and application techniques for minimizing interthread cache misses caused by destructive interference.

\subsection{Misses in a database workload}

We begin our investigation by analyzing per-segment misses for both OLTP and DSS workloads on an SMT processor. The results shown here were simulated on our 8-context SMT processor simulator described in Section 2 . For some experiments we simulate fewer than 8 contexts as well, to show the impact of varying the number of simultaneously-executing threads.

In the previous section we saw the individual miss rates for the four database memory regions, executing on a single-threaded uniprocessor. Table 4 shows the proportion of total misses due to each region, when executing on our 8-context SMT processor. From Table 4 , we see that, the PGA region is responsible for the majority of L1 and L2 misses. For example, the PGA accounts for $60 \%$ of the L1 misses and $98 \%$ of the L2 misses for OLTP (and 7\% and 58\% of total references to L1 and L2, respectively), making it the most important region for analysis. ${ }^{2}$

The PGA contains the per-process data (e.g., private stacks and local variables) that are used by each server process. PGA data is laid out in an identical fashion, i.e., at the same virtual addresses, in each process' address space. Furthermore, there are several hot spots in the PGA that are accessed throughout the life of each pro-

\footnotetext{
2. Note that the distribution of misses is skewed by the large number of conflict misses. When mapping conflicts are eliminated using the techniques described in the next section, the miss distribution changes substantially.
} 


\begin{tabular}{|l||l||r|l|r|r|}
\hline & Cache & $\begin{array}{c}\text { instruction } \\
\text { text }\end{array}$ & PGA & $\begin{array}{c}\text { SGA } \\
\text { buffer } \\
\text { cache }\end{array}$ & SGA-other \\
\hline \hline \multirow{2}{*}{ OLTP } & L1 & 28.6 & 60.0 & 0.9 & 10.5 \\
\cline { 2 - 6 } & L2 & 0.2 & 98.1 & 0.3 & 1.4 \\
\hline \multirow{2}{*}{ DSS } & L1 & 0.0 & 96.0 & 3.6 & 0.3 \\
\cline { 2 - 6 } & L2 & 0.0 & 99.9 & 0.1 & 0.0 \\
\hline
\end{tabular}

Table 4: Proportion of total misses (percent) due to each segment on an 8-context SMT. For the level 1 cache, we combined data and instruction misses.

cess. Consequently, SMT's fine-grained multithreading causes substantial destructive interference between the same virtual addresses in different processes. These conflicts also occur on single-threaded CPUs, but to a lesser extent, because context switching is much coarser grained than simultaneous-multithreaded instruction issue (PGA accounts for $71 \%$ of the misses on the singlethreaded CPU, compared to $84 \%$ on the 8-context SMT).

The SMT cache organization we simulate is a virtually-indexed/physically-tagged L1 cache with a physically-indexed/physically-tagged L2 cache. This structure is common for modern processors; it provides fast lookup for the L1 cache and ease of management for the L2 cache. Given this organization, techniques that alter the per-process virtual-address-space layout or the virtual-to-physical mapping could affect the miss rates for the L1 and L2 caches, respectively, particularly in the PGA. We therefore evaluate combinations of two software mechanisms that might reduce the high miss rates: virtual-to-physical page-mapping schemes and application-based, per-process virtual-address-space offsetting.

\subsection{Page-mapping policies}

Because the operating system chooses the mapping of virtual to physical pages when allocating physical memory, it plays a role in determining L2 cache conflicts. Operating systems generally divide physical memory page frames into colors (or bins); two physical pages have the same color if they index into the same location in the cache. By mapping two virtual pages to different colors, the page-mapping policy can eliminate cache conflicts between data on the two pages and improve cache performance [9].

The two most commonly-used page-mapping policies are page coloring and bin hopping. Page coloring exploits spatial locality by mapping consecutive virtual pages to consecutive physical page colors. IRIX, Solaris/SunOS and Windows NT augment this basic page coloring algorithm by either hashing the process ID with the virtual address or using a random seed for a process's initial page color. In contrast, Digital UNIX uses bin hopping, also known as first-touch. Bin hopping exploits temporal locality by cycling through page colors sequentially as it maps new virtual pages. Because page mappings are established based on reference order (rather than address- space order), pages that are mapped together in time will not conflict in the cache.

Our experiments indicate that, because multithreading magnifies the number of conflict misses, the page-mapping policy can have a large impact on cache performance on an SMT processor. Table 5 shows the L2 cache miss rates for OLTP and DSS workloads for various mapping schemes. The local miss rate is the number of L2 misses as a percentage of L2 references; the global miss rate is the ratio of $\mathrm{L} 2$ misses to total memory references. Bin hopping avoids mapping conflicts in the L2 cache most effectively, because it is likely to assign identical structures in different threads to non-conflicting physical pages. Consequently, miss rates are minuscule, and are stable across all numbers of hardware contexts, indicating that the OLTP and DSS "critical" working sets fit in a $16 \mathrm{MB}$ L2 cache. In contrast, page coloring follows the data memory layout; since this order is common to all threads (in the PGA), page coloring incurs more conflict misses, and increasingly so with more hardware contexts. In fact, at 4 contexts on DSS, almost all L2 cache references are misses. Hashing the process ID with the virtual address improves page coloring performance, but it still lags behind bin hopping.

Note that some of these conflict misses could also be addressed with higher degrees of associativity or with victim caching, but these solutions may either slow cache access times (associativity) or may have insufficient capacity to hold the large number of conflict misses in OLTP and DSS workloads (victim caches).

\subsection{Application-level offsetting}

Although effective page mapping reduces L2 cache conflicts, it does not impact on-chip L1 data caches that are virtually-indexed. In the PGA, in particular, identical virtual pages in the different processes will still conflict in the L1, independent of the physical page-mapping policy. One approach to improving the L1 miss rate is to "offset" the conflicting structures in the virtual address spaces of the different processes. For example, the starting virtual address of each newly-created process or segment could be shifted by (page size * process ID) bytes. This could be done manually in the application or by the loader.

Table 6 shows the L1 miss rates for the three pagemapping policies, both with and without address-space offsetting. The data indicate that using an offset reduced the L1 miss rate of all numbers of hardware contexts roughly to that of a wide-issue superscalar. Without offsetting, L1 miss rates doubled for OLTP and increased up to 12-fold for DSS, as the number of hardware contexts was increased to 8. Offsetting also reduced L2 miss rates for page coloring (data not shown). By shifting the virtual addresses, pages that would have been in the same bin under page coloring end up in different bins. 


\subsection{Constructive interference}

Simultaneous multithreading can exploit instruction sharing to improve instruction cache behavior, whether the instruction working set is large (OLTP) or small (DSS). In these workloads, each instruction block is touched by virtually all server threads, on average. The heavy instruction sharing generates constructive cache interference, as threads frequently prefetch instruction blocks for each other.

Each server thread for OLTP executes nearly identical code, because transactions are similar. A single-threaded superscalar cannot take advantage of this code sharing, because its threads are resident only on a coarse scheduling granularity. For example, a particular routine may be executed only near the beginning of a transaction. By the time the routine is re-executed by the same server process, the code has been kicked out of the cache. This occurs frequently, as the instruction cache is the largest performance bottleneck on these machines. On an 8-context SMT, however, the finer-grain multithreading increases the likelihood that a second process will re-execute a routine before it is replaced in the cache. This constructive cache interference reduces the instruction cache miss rate from $14 \%$ to $9 \%$, increasing processor throughput to the point where I/O latencies become the largest bottleneck, as discussed below.

Constructive interference does not require "lock-step" execution of the server threads. To the contrary, scheduling decisions and lock contention skew thread execution; for example, over the lifetime our 16 thread simulations, the "fastest" thread advances up to 15 transactions ahead of the "slowest" thread.

With DSS, the instruction cache hit rate is already almost $100 \%$ for one context, so constructive interference has no impact.

\subsection{Summary of multi-thread cache interference}

This section examined the effects of cache interference caused by fine-grained multithreaded instruction scheduling on an SMT processor. Our results, which are somewhat surprising, demonstrate that with appropriate page mapping and offsetting algorithms, an 8-context SMT processor can maintain L1 and L2 cache miss rates roughly commensurate with the rates for a singlethreaded superscalar. Even for a less aggressive memory configuration than the one we normally simulate (e.g., 64KB instruction cache, 32KB data caches and 4MB L2 caches), destructive interference remains low. Only when the $\mathrm{L} 2$ cache size is as low as $2 \mathrm{MB}$ - conservative even for today's database servers - does inter-thread interference have an impact. We have also shown that constructive interference in the I-cache benefits performance on the SMT relative to a traditional superscalar.

Overall, with proper software-mapping policies, the cache behavior for database workloads on SMT processors is roughly comparable to conventional processors. In both cases, however, the absolute miss rates are high and will still cause substantial stall time for executing processes. Therefore, the remaining question is whether SMT's latency-tolerant architecture can absorb that stall

\begin{tabular}{|c|c|c|c|c|c|c|c|c|c|}
\hline \multirow{3}{*}{$\begin{array}{l}\text { Page-mapping } \\
\text { technique }\end{array}$} & \multirow{3}{*}{$\begin{array}{l}\text { Type of } \\
\text { L2 miss } \\
\text { rate }\end{array}$} & \multicolumn{4}{|c|}{ OLTP } & \multicolumn{4}{|c|}{ DSS } \\
\hline & & \multicolumn{4}{|c|}{ Number of contexts } & \multicolumn{4}{|c|}{ Number of contexts } \\
\hline & & 1 & 2 & 4 & 8 & 1 & 2 & 4 & 8 \\
\hline \multirow[t]{2}{*}{ Bin hopping } & global & 0.3 & 0.3 & 0.3 & 0.3 & 0.0 & 0.0 & 0.0 & 0.0 \\
\hline & local & 2.7 & 2.7 & 2.6 & 2.4 & 5.3 & 4.4 & 0.4 & 0.3 \\
\hline \multirow{2}{*}{ Page coloring } & global & 3.4 & 3.5 & 5.1 & 6.7 & 0.3 & 0.3 & 6.6 & 9.1 \\
\hline & local & 34.4 & 38.0 & 50.3 & 58.9 & 39.9 & 41.6 & 94.8 & 96.1 \\
\hline \multirow{2}{*}{$\begin{array}{l}\text { Page coloring with pro- } \\
\text { cess id hash }\end{array}$} & global & 1.8 & 1.6 & 1.4 & 1.2 & 0.2 & 0.2 & 0.2 & 0.2 \\
\hline & local & 17.3 & 16.1 & 12.0 & 8.7 & 32.5 & 28.1 & 2.7 & 2.1 \\
\hline
\end{tabular}

Table 5: Global and local L2 cache miss rates (in percentages) for 16 threads running on an SMT with 1-8 contexts. Note that the local miss rates can be skewed by the large number of $L 1$ conflict misses (as shown in the next table). For example, the $0.3 \%$ local miss rate (bin hopping, 8 contexts) is much lower than that found for typical DSS workloads.

\begin{tabular}{|c|c|c|c|c|c|c|c|c|c|}
\hline \multirow{3}{*}{$\begin{array}{l}\text { Page-mapping } \\
\text { technique }\end{array}$} & \multirow{3}{*}{$\begin{array}{c}\text { Application } \\
\text { offsetting }\end{array}$} & \multicolumn{4}{|c|}{ OLTP } & \multicolumn{4}{|c|}{ DSS } \\
\hline & & \multicolumn{4}{|c|}{ Number of contexts } & \multicolumn{4}{|c|}{ Number of contexts } \\
\hline & & 1 & 2 & 4 & 8 & 1 & 2 & 4 & 8 \\
\hline$\overline{\text { Bin hopping }}$ & no offset & $\overline{8.2}$ & $\overline{8.9}$ & $\overline{12.3}$ & $\overline{16.0}$ & $\overline{1.2}$ & $\overline{1.4}$ & $\overline{15.0}$ & $\overline{18.8}$ \\
\hline & offset & 8.4 & 8.5 & 8.6 & 8.7 & 1.2 & 1.3 & 1.6 & 2.0 \\
\hline Page coloring & no offset & 7.9 & 8.6 & 12.5 & 17.0 & 1.2 & 1.3 & 17.7 & 25.7 \\
\hline & offset & 8.3 & 8.5 & 8.7 & 8.8 & 1.2 & 1.3 & 1.6 & 2.2 \\
\hline Page coloring with & no offset & 8.1 & 8.9 & 12.9 & 18.5 & 1.2 & 1.4 & 15.0 & 19.3 \\
\hline process id hash & offset & 8.4 & 8.7 & 8.9 & 9.1 & 1.2 & 1.3 & 1.5 & 2.2 \\
\hline
\end{tabular}

Table 6: Local L1 cache miss rates (in percentages) for 16 threads running on an SMT, with and without offsetting of perprocess PGA data. For these experiments, an offset of $8 \mathrm{~KB}$ * thread ID as used. 

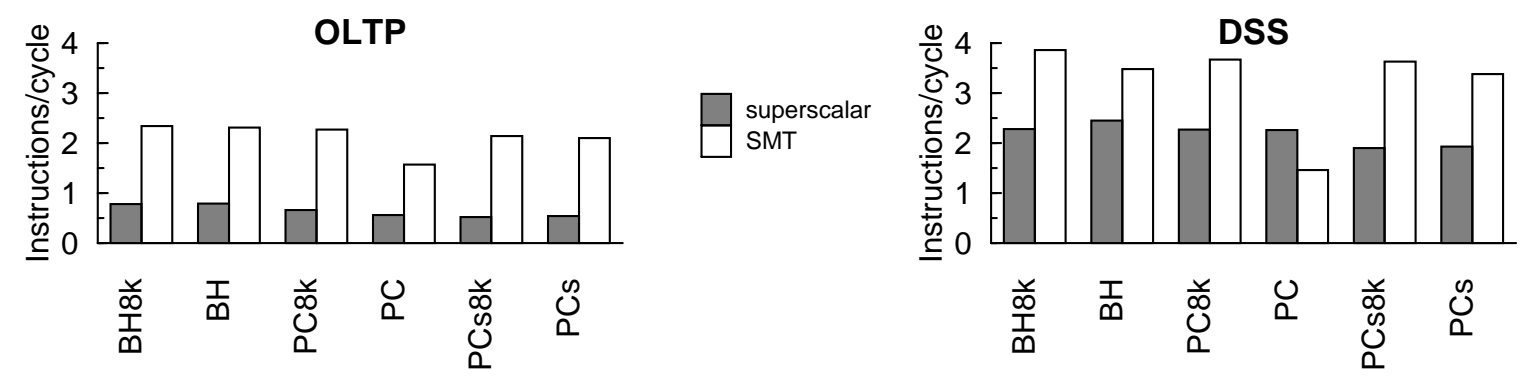

Figure 3. Comparison of throughput for various page-mapping schemes on a superscalar and 8-context SMT. The bars compare bin hopping $(\mathrm{BH})$, page coloring $(\mathrm{PC})$, and page coloring with an initial random seed (PCs), with (8k) and without virtual address offsets.

time, providing an increase in overall performance. This is the subject of the following section.

\section{SMT performance on database workloads}

This section presents the performance of OLTP and DSS workloads on an SMT processor, compared to a single-threaded superscalar. We compare the various software algorithms for page coloring and offsetting with respect to their impact on instruction throughput, measured in instructions per cycle. The results tell us that SMT is very effective for executing database workloads.

Figure 3 compares instruction throughput of SMT and a single-threaded superscalar for the alternative pagemapping schemes, both with and without address offsets. From this data we draw several conclusions. First, although the combination of bin hopping and application offsetting provides the best instruction throughput (2.3 IPC for OLTP, 3.9 for DSS) on an 8-wide SMT, several other alternatives are close behind. The marginal performance differences give designers flexibility in configuring SMT systems: if the DBMS provides offsetting in the PGA, the operating system has more leeway in its choice of page-mapping algorithms; alternatively, if an application does not support offsetting, bin hopping can be used alone to obtain almost comparable performance.

Second, with either bin hopping or any of the pagemapping schemes with offsetting, the OLTP and DSS "critical" working sets fit in the SMT cache hierarchy, thereby reducing destructive interference. Using these techniques, SMT achieves miss rates nearly as low as those of a single-threaded superscalar for all numbers of hardware contexts.

Third, it is clear from Figure 3 that SMT is highly effective in tolerating the high miss rates of this workload, providing a substantial throughput improvement over the superscalar. For DSS, for example, the best SMT policy (BH8k) achieves a $57 \%$ performance improvement over the best superscalar scheme (BH). Even more impressive, for the memory-bound OLTP, the SMT processor shows a $200 \%$ improvement in utilization over the superscalar (BH8k for both cases).

Table 7 provides additional architectural insight into the large increases in IPC, focusing on SMT's ability to hide instruction and data cache misses, as well as branch mispredictions. The comparison of the average number of outstanding D-cache misses illustrates SMT's effectiveness at hiding data cache miss latencies. For OLTP, SMT shows a 3-fold increase (over the superscalar) in the amount of memory system parallelism, while DSS shows a 1.5-fold improvement. Since memory latency is more important than memory bandwidth in these workloads, increased memory parallelism translates to greater processor throughput.

Simultaneous multithreading also addresses fetching bottlenecks resulting from branch mispredictions and instruction cache misses. The superscalar fetches 50\% and $100 \%$ more wrong-path (i.e., wasted) instructions than SMT for OLTP and DSS, respectively. By interleaving instructions from multiple threads, and by choosing to fetch from threads that are making the most effective utilization of the execution resources [23], SMT reduces the need for (and more importantly, the cost of) speculative execution [10]. SMT also greatly reduces the number of cycles in which no instructions can be fetched due to misfetches or I-cache misses. On the DSS workload SMT nearly eliminates all zero-fetch cycles. On OLTP, fetch stalls are reduced by $78 \%$; zero-fetch cycles are still $15.5 \%$, because OLTP instruction cache miss rates are higher.

Finally, the last two metrics illustrate instruction issue effectiveness. The first is the number of cycles in which no instructions could be issued: SMT reduces the number of zero-issue cycles by $68 \%$ and $93 \%$ for OLTP and

\begin{tabular}{|l||r|r|r|r|}
\hline \multicolumn{1}{|c||}{ Metric } & \multicolumn{2}{c|}{ OLTP } & \multicolumn{2}{c|}{ DSS } \\
\cline { 2 - 5 } & \multicolumn{1}{|c|}{ SS } & \multicolumn{1}{c|}{ SMT } & \multicolumn{1}{c|}{ SS } & \multicolumn{1}{c|}{ SMT } \\
\hline \hline $\begin{array}{l}\text { Avg. \# of outstanding D- } \\
\text { cache misses }\end{array}$ & 0.66 & 2.08 & 0.48 & 0.75 \\
\hline $\begin{array}{l}\text { Wrong-path instructions } \\
\text { fetched (\%) }\end{array}$ & 60.0 & 40.0 & 20.7 & 9.9 \\
\hline Zero-fetch cycles (\%) & 55.4 & 15.5 & 29.6 & 1.8 \\
\hline Zero-issue cycles (\%) & 57.5 & 18.5 & 34.9 & 2.3 \\
\hline 6-issue cycles (\%) & 8.6 & 32.8 & 22.4 & 58.6 \\
\hline
\end{tabular}

Table 7: Architectural metrics for superscalar (SS) and 8context SMT on OLTP and DSS workloads. 
DSS, respectively. The second is the percentage of cycles in which the two architectures successfully issued 6 instructions (the maximum possible for this all-integer workload). Not surprisingly, SMT has a huge advantage over the superscalar in finding 6 instructions to issue, showing a 3.8-fold increase for OLTP and a 2.6-fold gain for DSS.

Overall, these results demonstrate that simultaneous multithreading can tolerate memory latencies, exploit inter-thread instruction sharing, and limit inter-thread interference on memory-intensive database workloads. The 3-fold throughput improvement for the memorybound OLTP workload, in particular, shows that SMT's latency tolerance makes SMT an extremely strong candidate architecture for future database servers.

\section{Related work}

We are aware of only one other study that has examined the performance of commercial workloads on multithreaded architectures. Eickemeyer, et al., [5] used trace-driven simulation to evaluate the benefits of coarsegrain multithreading for TPC-C on the OS/400 database. By using two or three threads, throughput increased by $70 \%$; but with more than 3 threads, no further gains were achieved. Because their coarse-grain architecture only switched threads on second-level cache misses, multithreading could not hide L1 instruction and data cache misses, which are very common in commercial workloads. In contrast, simultaneous multithreading interleaves multiple threads on a cycle-by-cycle basis. Therefore it hides latencies more effectively, and can utilize more hardware contexts to achieve greater throughput gains.

While several other studies characterized memorysystem behavior of database workloads, only one study analyzed memory access patterns in detail. Trancoso, et al., [22] used a public-domain DBMS (Postgres95) to examine the memory access patterns of several DSS queries on cache-coherent shared-memory multiprocessors, and contrasted the cache effects on various data structures in the Postgres95 DBMS. Our study does not examine individual data structures, but contrasts the effects of OLTP and DSS workloads on the behavior of database memory regions in a widely-used commercial database application.

Our paper also extends the cache behavior analysis presented in other prior work. Franklin, et al., [6] identified the scarcity of loops and context switches as contributors to high instruction cache miss rates in commercial applications. Maynard, et al., [12] highlighted the large instruction footprints and high instruction cache miss rates of OLTP workloads. In another study, Cvetanovic and Bhandarkar [3] used performance counters on the DEC Alpha chip family (21064 and 21164) to identify the performance characteristics of a range of applications, including two commercial workloads.
In addition to characterizing database memory behavior, prior research has also identified other bottlenecks, such as pin bandwidth and I/O, in OLTP workloads. Perl and Sites [14] demonstrated that both high bandwidth and low latency are required to effectively run OLTP and other commercial applications. Their experiments assumed single-cycle instruction latencies and on-chip cache sizes of the Alpha 21064 (8KB I, 8KB D) and 21164 (8KB I, 8KB D, and 96KB unified on-chip L2). In our experiments, we model a more aggressive future processor, with larger on-chip caches and a higher bandwidth L1-L2 bus (256-bit bus with 1 bus transfer per processor clock). Pin bandwidth was therefore a smaller bottleneck; the larger caches and constructive instruction cache interference (due to SMT) also reduced the bandwidth demands.

Rosenblum, et al., [16] found that both CPU idle time and kernel activity were significant when running an OLTP workload on Sybase. CPU idle time was greater than $30 \%$ because of disk $\mathrm{I} / \mathrm{O}$; kernel activity accounted for $38 \%$ of non-idle execution time. However, their configuration had only one server process to handle 20 clients. Our experiments showed that idle time and kernel activity can both be reduced with a well-tuned commercial DBMS. For example, I/O latencies can be hidden more effectively by using more server processes (as is typically done in audit runs of TPC benchmarks).

Finally, a few studies have used OLTP and DSS workloads to evaluate multiprocessor systems or investigate other performance issues. Thakkar and Sweiger [18] identified disk I/O and the system bus as bottlenecks for the TP1 benchmark on a Sequent Symmetry. Lovett and Clapp [11] focused on the L2 cache behavior and scalability of the Sequent STiNG CCNUMA multiprocessor. Verghese, et al., [24] evaluated page migration and replication for CC-NUMA architectures. Torrellas, et al., [19] used Oracle running TP1 to characterize OS cache activity. Kawaf, et al., [8] and Piantedosi, et al., [15] described optimizations for improving TPC-C performance on Digital AlphaServers.

\section{Conclusions}

This paper explored the behavior of database workloads on simultaneous multithreaded processors, concentrating in particular on the challenges presented to the memory system. For our study, we collected traces of the Oracle DBMS executing under Digital Unix on DEC Alpha processors, and processed those traces with simulators of wide-issue superscalar and simultaneous multithreaded processors.

The conclusions from our simulation study are threefold. First, while database workloads have large footprints, there is substantial reuse that results in a small, cacheable "critical" working set, if cache conflicts can be minimized. Second, while cache conflicts are high, particularly in the per-process private data region of the 
database, they often result from poorly-suited mapping policies in the operating system. By selecting the appropriate mapping policy, and using application-based address offsetting, conflict misses on an 8-context SMT can be reduced to the point where they are roughly commensurate with miss rates for a superscalar. Third, once cache conflicts are reduced to the superscalar level, an SMT processor can achieve substantial performance gains over a superscalar with similar resources. We show a 3-fold improvement in utilization (IPC) for the OLTP workload and a 1.5-fold increase for DSS. These performance gains occur because of SMT's ability to tolerate latencies through fine-grained sharing of all processor resources among the executing threads; this is demonstrated by our detailed measurements indicating that SMT achieves a striking improvement in memory-system utilization and fetch and issue efficiency, when compared with the single-threaded superscalar.

We believe that these results indicate that SMT's latency tolerance makes it an excellent architecture for database servers.

\section{Acknowledgments}

We would like to thank Marco Annaratone from DEC WRL for supporting this work, Jef Kennedy from Oracle for reviewing the manuscript, and Drew Kramer and Parthasarathy Ranganathan for their technical assistance in the research. We would also like to thank the referees, whose comments strengthened this paper. Eggers' and Levy's research are supported by the DEC External Research Program, NSF grants MIP-9632977 and CCR9632769, DARPA contract F30602-97-2-0226, ONR grant N00014-96-1-0402, and the Washington Technology Center. Lo was supported by an Intel Graduate Fellowship, Lo and Parekh by DEC WRL summer internships.

\section{References}

[1] J. Anderson, et al. Continuous profiling: Where have all the cycles gone? In 16th ACM Symp. on Operating System Principles, p. 1-14, Oct. 1997.

[2] L. A. Barroso, et al. Memory system characterization of commercial workloads. In 25th Ann. Int'l Symp. on Computer Arch., June 1998.

[3] Z. Cvetanovic and D. Bhandarkar. Characterization of Alpha AXP performance using TP and SPEC workloads. In 21st Ann. Int'l Symp. on Computer Arch., p. 60-70, April 1994.

[4] S. Eggers, et al. Simultaneous multithreading: A platform for next-generation processors. In IEEE Micro, p. 12-19, Oct. 1997.

[5] R. Eickemeyer, et al. Evaluation of multithreaded uniprocessors for commercial application environments. In 23rd Ann. Int'l Symp. on Computer Arch., p. 203-212, May 1996.

[6] M. Franklin, et al. Commercial workload performance in the IBM POWER2 RISC System/6000 processor. IBM J. of Research and Development, 38(5):555-561, April 1994.

[7] V. Gokhale. Design of the 64-bit option for the Oracle7 rela- tional database management system. Digital Technical Journal, 8(4):76-82, 1996.

[8] T. Kawaf, et al. Performance analysis using very large memory on the 64-bit AlphaServer system. Digital Technical Journal, 8(3):58-65, 1996.

[9] R. Kessler and M. Hill. Page placement algorithms for large real-indexed caches. ACM Trans. on Computer and Systems, 10(4):338-359, November 1992.

[10] J. Lo, et al. Tuning compiler optimizations for simultaneous multithreading. In 30th Int'l Symp. on Microarchitecture, $\mathrm{p}$. 114-124, December 1997.

[11] T. Lovett and R. Clapp. STiNG: A CC-NUMA computer system for the commercial marketplace. In 23rd Ann. Int'l Symp. on Computer Arch., p. 308-317, May 1996.

[12] A. M. Maynard, et al. Contrasting characteristics and cache performance of technical and multi-user commercial workloads. In Sixth Int'l Conference on Arch. Support for Prog. Lang. and Operating Systems, p. 145-156, Oct. 1994.

[13] S. McFarling. Combining branch predictors. Technical Report TN-36, DEC-WRL, June 1993.

[14] S. Perl and R. Sites. Studies of Windows NT performance using dynamic execution traces. In Second USENIX Symp. on Operating System Design and Implementation, p. 169-183, Oct. 1996.

[15] J. Piantedosi, et al. Performance of TruCluster systems under the TPC-C benchmark. Digital Technical Journal, 8(3):4657, 1996.

[16] M. Rosenblum, et al. The impact of architectural trends on operating system performance. In 15th ACM Symp. on Operating System Principles, p. 285-298, December 1995.

[17] A. Srivastava and A. Eustace. ATOM: A system for building customized program analysis tools. In ACM SIGPLAN '94 Conference on Programming Language Design and Implementation, p. 196-205, June 1994.

[18] S. Thakkar and M. Sweiger. Performance of an OLTP application on Symmetry multiprocessor system. In 17th Ann. Int'l Symp. on Computer Arch., p. 228-238, May 1990.

[19] J. Torrellas, et al. Characterizing the caching and synchronization performance of a multiprocessor operating system. In Fifth Int'l Conference on Arch. Support for Prog. Lang. and Operating Systems, p. 162-174, Oct. 1992.

[20] Transaction Processing Performance Council. TPC Benchmark B Standard Specification Revision 2.0. June 1994.

[21] Transaction Processing Performance Council. TPC Benchmark D (Decision Support) Standard Specification Revision 1.2. November 1996.

[22] P. Trancoso, et al. The memory performance of DSS commercial workloads in shared-memory multiprocessors. In $3 r d$ Ann. Int'l Symp. on High-Performance Computer Architecture, p. 250-260, February 1997.

[23] D. Tullsen, et al. Exploiting choice: Instruction fetch and issue on an implementable simultaneous multithreading processor. In 23rd Ann. Int'l Symp. on Computer Arch., p. 191202, May 1996.

[24] B. Verghese, et al. Operating system support for improving data locality on CC-NUMA compute servers. In Seventh Int'l Conference on Arch. Support for Prog. Lang. and Operating Systems, p. 279-289, Oct. 1996. 